SLAC-PUB-11829

April 2006

\title{
Wakefields of Sub-Picosecond Electron Bunches a
}

\author{
Karl L.F. Bane \\ Stanford Linear Accelerator Center, Stanford University, \\ Stanford, CA 94309 USA
}

\author{
Invited talk at the \\ Workshop on the Physics and Applications of High \\ Brightness Electron Beams (PAHBEB 2005) \\ Erice, Sicily, Italy \\ October 9-14, 2005
}

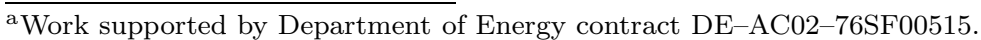




\title{
WAKEFIELDS OF SUB-PICOSECOND ELECTRON BUNCHES*
}

\author{
K. L. F. BANE \\ Stanford Linear Accelerator Center, \\ 2575 Sand Hill Road, \\ Menlo Park, CA 94025, USA \\ E-mail: kbane@slac.stanford.edu
}

\begin{abstract}
We discuss wakefields excited by short bunches in accelerators. In particular, we review some of what has been learned in recent years concerning diffraction wakes, roughness impedance, coherent synchrotron radiation wakes, and the resistive wall wake, focusing on analytical solutions where possible. As examples, we apply formulas for these wakes to various parts of the Linac Coherent Light Source (LCLS) project. The longitudinal accelerator structure wake of the SLAC linac is an important ingredient in the LCLS bunch compression process. Of the wakes in the undulator region, the dominant one is the resistive wall wake of the beam pipe.
\end{abstract}

\section{Introduction}

In recent accelerator projects one often finds high energy bunches of electrons or positrons that are simultaneously short, intense, and have small emittances. In the International Linear Collider (ILC) trains of $3 \mathrm{nC}$, $300 \mu \mathrm{m}$ long bunches are accelerated through $10 \mathrm{~km}$ of linac, on their way the final focus and the collision point. In the Linac Coherent Light Source (LCLS), a SASE FEL, a $1 \mathrm{nC}$ bunch is compressed to a length of $20 \mu \mathrm{m}$, accelerated in $500 \mathrm{~m}$ of linac, before entering the undulator for lasing ${ }^{1}$. In both cases a major challenge is keeping in check wakefields that are induced in various parts of the accelerator and that tend to increase emittances, thereby degrading luminosity (in the former case) or lasing (in the latter).

In August 1987 a workshop entitled "Impedance Beyond Cut-Off" was held at the Lawrence Berkeley Laboratory. In the foreword to the proceedings to this workshop, the editor noted that "latest designs of many ad-

*This work is supported by the Department of Energy, contract DE-AC02-76SF00515. 
vanced accelerator systems ... involve charged particle beams of extremely short duration in time (a few tens of picoseconds and shorter)" ${ }^{2}$. Nowadays, a similar statement can still be made, but with the words "tens of picoseconds" probably changed to "hundreds of femtoseconds." Over the intervening years, as projects have been calling for ever shorter bunches the understanding of short-bunch wake/high frequency impedance has also been increasing. This report is a review of some of what has been learned about this subject in recent years.

One way of measuring "short" is to take the ratio $\sigma_{z} / a$, where $\sigma_{z}$ is the rms bunch length and $a$ is beam pipe radius. According to this simple criterion both the ILC bunch in the main linac and the LCLS bunch in the final linac are short $\left(\sigma_{z} / a=0.009\right.$ in both cases). In general, we can say that as bunches become shorter the longitudinal wakefields become stronger and the transverse wakes weaker. As bunches become shorter/their frequency content becomes higher, new sources of wakefields become important- e.g. the roughness wake - and familiar wakefields display unfamiliar behavior-e.g. the resistive wall wake. Numerically calculating a short-range wake/high frequency impedance can be a difficult task. Often, however, analytical approximations exist-especially for cylindrically symmetric structures - that can still be accurate. One factor that helps is that the broad-band (averaged) impedance suffices, since the (broad) bunch spectrum smooths out resonances.

In this report we present in some detail wakes that become important for short bunches, including diffraction wakes, roughness impedance, coherent synchrotron radiation (CSR) wake, and the resistive wall wake. We focus on analytical solutions, and on longitudinal over transverse wakes. Formulas presented here can be used to set up an impedance budget, to order in importance the various wake sources in a project. However, for some objects, especially $3 \mathrm{D}$ objects, numerical calculations will still be necessary.

\subsection{The LCLS}

As concrete examples for the wake formulas, throughout the text we will consider parts of the LCLS project. Figure 1 gives a schematic of the LCLS, displaying important machine parameters and the beam properties of energy, rms bunch length, rms relative energy spread $\left(\sigma_{\delta}\right)$, at various locations. The LCLS comprises an rf gun, four S-band accelerator regions (Linacs-0 to -3), an X-band structure, two chicane bunch compressors (named $\mathrm{BC} 1$ and $\mathrm{BC} 2$ ), and an undulator. The bunch charge is 
$e N=1 \mathrm{nC}$. At the end of $\mathrm{BC} 1$, at energy $E=250 \mathrm{MeV}$, the bunch shape is roughly Gaussian, with rms length $\sigma_{z}=0.19 \mathrm{~mm}$. At the end of $\mathrm{BC} 2$, $E=4.54 \mathrm{GeV}$. Beginning at this location the rms length $\sigma_{z}=22 \mu \mathrm{m}$; the bunch shape, however, is "double horned"-it consists of a $3 \mathrm{kA}$ flat-top, with leading and trailing spikes 12-16 kA high, each of rms length $\sim 2 \mu \mathrm{m}$. The final energy is $14.1 \mathrm{GeV}$.

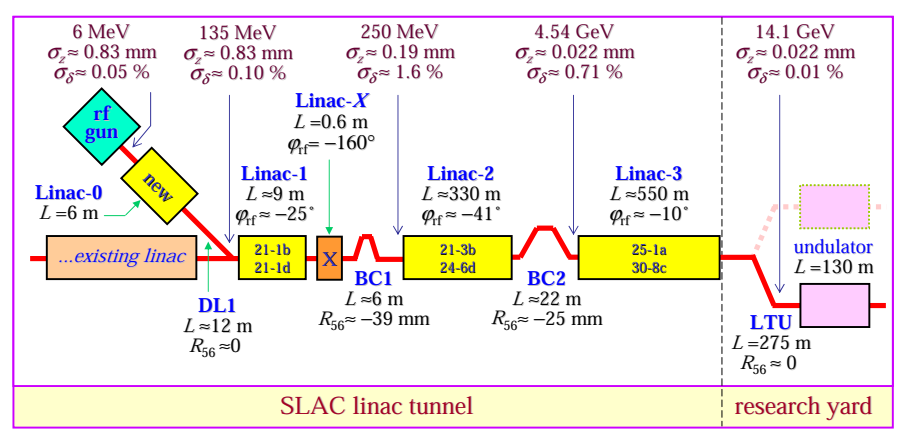

Figure 1. Schematic of the LCLS.

In the LCLS the longitudinal wakes tend to be more important than the transverse wakes. Also, in a SASE FEL, like the LCLS, we are not sensitive to projected emittance; amplification is sensitive to slice properties $(\sim 1 \mu \mathrm{m}$ scale vs. a bunch rms of $20 \mu \mathrm{m}$ ). Wakefield effects, however, are head-tail effects, and only weakly affect slice properties directly. Nevertheless, in order not to interfere with lasing, there is a limit in allowable induced energy change in the undulator, to be less than a few times the Pierce parameter $(\lesssim 0.3 \%)$. In addition, there are bunch specifications on bandwidth $(<$ $0.1 \%)$, and on slice alignment $\left(<\sigma_{x}\right)$, with $\sigma_{x}$ transverse rms beam size.

In this report, for examples, we consider the effects of the accelerator structure wake in the linacs, the CSR wake in the BC2 chicane, and the wakes of transitions, roughness, and resistive wall in the beam pipe of the undulator. Note that in the linacs the wakes are an integral part of the compression process that determines the final phase space of the beam. In the chicane, CSR wake induced energy change results, through dispersion, in emittance growth. In the undulator region, the resistive wall wake of the $130 \mathrm{~m}$ beam pipe dominates; originally the beam pipe was meant to be round with $a=2.5 \mathrm{~mm}$, made of stainless steel coated with copper. 


\section{WAKES AND IMPEDANCES}

We begin by reviewing basic properties of wakes and impedances. The reader is referred to A. Chao's book for more details ${ }^{3}$.

Consider a point particle of unit charge moving at the speed of light $c$ through a structure, that is followed, at distance $s$, by a test particle, that is also moving at $c$. The longitudinal wake $W(s)$ is the voltage loss experienced by the test particle, typically given in units $[\mathrm{V} / \mathrm{C}]$ for a single structure, in $[\mathrm{V} / \mathrm{C} / \mathrm{m}]$ for a periodic one. The wake is zero if the test particle is in front $(s<0)$. For a bunch of longitudinal charge distribution $\lambda_{z}$, the bunch wake $\mathcal{W}(s)$ - the voltage gain for a test particle at position $s$-is given by

$$
\mathcal{W}(s)=-\int_{0}^{\infty} W\left(s^{\prime}\right) \lambda_{z}\left(s-s^{\prime}\right) d s^{\prime}
$$

The average of minus the bunch wake, $-\langle\mathcal{W}\rangle$, gives the loss factor; the rms $\mathcal{W}_{r m s}$ gives the energy spread increase: $\Delta E_{r m s}=e N L \mathcal{W}_{r m s}$, with $L$ the length of structure (in the periodic case).

The impedance is the Fourier transform of the wake:

$$
Z(k)=\frac{1}{c} \int_{0}^{\infty} W(s) e^{i k s} d s,
$$

with $k$ the wave number. In a cylindrically symmetric structure the impedance/wakes excited by a particle off-axis vary as $\cos m \theta$, with $m$ an integer and $\theta$ the azimuthal angle; the dominant transverse wakefield is the dipole $(m=1)$ wake. Here the transverse wake $W_{x}$ is defined as the dipole force experienced by the test particle per unit offset of the driving particle, given in $\left[\mathrm{V} / \mathrm{C} / \mathrm{m}^{2}\right]$ in the periodic case. The transverse impedance $Z_{x}$ is, by convention, taken to be $i$ times the Fourier transform of $W_{x}$. For the special case of the resistive wall wake the longitudinal $(m=0)$ and transverse $(m=1)$ wakes and impedances are related by

$$
W_{x}(s)=\frac{2}{a^{2}} \int_{0}^{s} W\left(s^{\prime}\right) d s^{\prime}, \quad Z_{x}(k)=\frac{2}{a^{2} k} Z(k) .
$$

These relations, as we will see, hold also for many of the short-range wakes/high-frequency impedances considered in this report, though they are not, in general, valid.

We sometimes denote an impedance/wake as being resistive, inductive, or capacitive, from analogy to simple circuit element properties. By these labels we mean objects for which $\mathcal{W}(s)$ is proportional to $\lambda_{z}(s), \lambda_{z}^{\prime}(s)$, 
and $\int_{-\infty}^{s} \lambda_{z}\left(s^{\prime}\right) d s^{\prime}$, respectively. Note that these imply that $W(s)$ is proportional to, respectively, $\delta(s), \delta^{\prime}(s)$, and $H(s)$, with $H(s)$ the unit step function ( $=0$ for $s<0 ; 1$ for $s>0$ ).

\subsubsection{Asymptotic Behavior of $Z(k), W(s)$, and $\mathcal{W}(s)$ (see e.g. [3])}

As will be seen in the following section, in some cases the asymptotic behavior of the wake $W(s)$ or the impedance $Z(k)$ is not integrable. We find e.g. asymptotic wakes of the form $W \sim-A s^{-\alpha}$, with $1<\alpha<2$ and $A$ a constant (an example is the longitudinal, long-range resistive-wall wake). In such cases it appears that one cannot simply convert between asymptotic forms of $W$ and $Z$, or from the asymptotic form of $W$ to that of $\mathcal{W}$. However, we know that such asymptotic behavior cannot continue forever, and both $W$ and $Z$ are always integrable: $\int_{-\infty}^{\infty} W(s) d s=Z(0)=0$ and $\int_{-\infty}^{\infty} Z(k) d k=2 \pi W(0)$ is finite (at least cut-off by finite beam energy). This knowledge allows us to make such conversions, either through differentiation under the integral sign or through integration by parts.

For example, consider the asymptotic wake form discussed above. The asymptotic behavior of the bunch wake can be obtained using integration by parts (we need to assume the bunch shape is smooth):

$$
\mathcal{W}(s)=\frac{A}{\alpha-1} \int_{0}^{\infty} \frac{d \lambda_{z}(s-z)}{d z} \frac{d z}{z^{\alpha-1}} .
$$

We see that knowledge of the very short-range wake, how it behaves as $s \rightarrow 0$, is not necessary.

\subsection{Considerations for Short Bunches}

Considerations especially relevant for short bunches are:

Catch-up distance: In calculating the effect on a beam, the wake is typically taken to act instantaneously. For very short bunches there can be a significant lag between the generation of radiation by the head of a bunch and its effect on tail particles. When a head particle passes a vacuum chamber object, such as the beginning of a cavity, that information cannot arrive at a tail particle until a downstream distance $z=a^{2} / 2 s$, where $a$ is the beam pipe radius and $s$ is the distance between the two particles. For example, if $a=1 \mathrm{~cm}$ and $s=20 \mu \mathrm{m}$, then the catch-up distance is $2.5 \mathrm{~m}$.

Transients: Similarly, for periodic structures, the interaction with a short bunch will entail an initial transient region before the steady-state wake is reached (see the example shown in Fig. 2). For a Gaussian bunch 
with length $\sigma_{z}$ the transient regime will last $z \sim a^{2} / 2 \sigma_{z}$. As a bunch becomes shorter, the transients become ever more important.

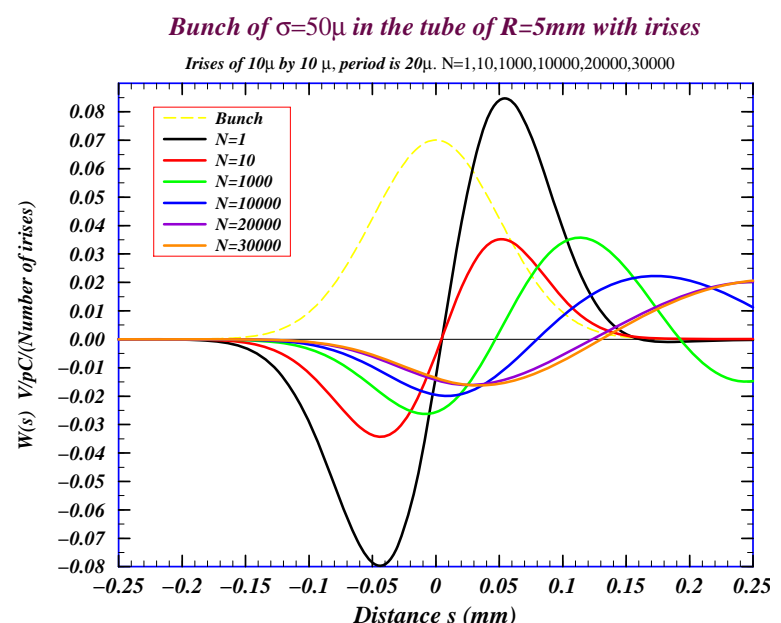

Figure 2. Simulation of wake per period generated by a bunch in a tube with $N$ small corrugations, showing the passage from transient to steady-state behavior; the bunch shape is given in yellow (from Ref. [5]).

Limiting value of wake: For periodic, cylindrically symmetric structures whose closest approach to the axis is $a$, the steady state wakes have the property

$$
W\left(0^{+}\right)=\frac{Z_{0} c}{\pi a^{2}} \quad \text { and } \quad W_{x}^{\prime}\left(0^{+}\right)=\frac{2 Z_{0} c}{\pi a^{4}},
$$

with $W_{x}\left(0^{+}\right)=0$, where $Z_{0}=377 \Omega$. This is true for a resistive pipe ${ }^{3}$, a disk-loaded accelerator structure ${ }^{4}$, a pipe with small periodic corrugations ${ }^{5,6}$, and a dielectric tube within a pipe ${ }^{7}$; it seems safe to assume that it is generally true. For a non-round structure the result will be a different constant $\left[\right.$ e.g. for 2 parallel, resistive plates separated by $2 a, W\left(0^{+}\right)=$ $\pi Z_{0} c / 16 a^{2}{ }^{8}$, but again a constant dependent on transverse dimensions and independent of material properties. Note that these formulas give an upper limit to the rate of energy loss and to the dipole wake that can be induced by a short bunch.

Finite energy: The impedance will drop sharply to zero for frequencies $k>\gamma / a$, with $\gamma$ the Lorentz energy factor. In the time domain when 
$\sigma_{z}<a / \gamma, \sigma_{z}$ should be replaced by $a / \gamma$ in wakefield formulas. For example, if $a=1 \mathrm{~cm}$ and energy $E=14 \mathrm{GeV}$ this occurs when $\sigma_{z}=0.4 \mu \mathrm{m}$.

\section{TYPES OF SHORT BUNCH WAKES}

We discuss four types of wakes that tend to be important for short bunches: diffraction wakes, roughness impedance, coherent synchrotron radiation wakes, and resistive wall wakes. In all cases the structures considered are cylindrically symmetric with a beam pipe radius $a$. Impedances presented here are valid at high frequencies, wakes at short distance. Note that for all expressions of (point charge) wakes given, it is implied that they are multiplied by the unit step function $H(s)$.

\subsection{Diffraction Wakes}

When a short bunch $\left(\sigma_{z} / a \ll 1\right)$ passes by an abrupt change in the beam pipe, such as at the beginning of a cavity, waves begin to radiate from the corner in a manner that can be described by the diffraction theory of light. If the corner is part of a shallow step or shallow cavity Fraunhoffer diffraction applies, if it is part of a deep cavity Fresnel diffraction applies. A third important example, a periodic array of deep cavities, is a model for a multi-cell (disk-loaded) accelerator structure.

\subsubsection{Steps and Cavities}

Consider beam pipes of radius $a$ and $b$ with $b>a$. For an abrupt step connecting the pipes, Heifets and Kheifets have shown that the high frequency impedance $Z \approx Z_{0} \ln (b / a) / \pi\left(Z_{0}=377 \Omega\right)$ when moving from the pipe of radius $a$ to that that of radius $b$, and $Z \approx 0$ when moving from $b$ to $a^{9}$. Combining the two cases (in either order) to make a pair of shallow transitions or a shallow cavity (see Fig. 3a), they obtain for impedance, $Z=Z_{0} \ln (b / a) / \pi$, implying a resistive short-range wake:

$$
W(s)=\frac{Z_{0} c}{\pi} \ln (b / a) \delta(s) .
$$

For the transverse case the impedance, $Z_{x}=Z_{0}\left(a^{-2}-b^{-2}\right) /(\pi k)$, and the wake is capacitive ${ }^{10}$ :

$$
W_{x}(s)=\frac{Z_{0} c}{\pi}\left(\frac{1}{a^{2}}-\frac{1}{b^{2}}\right) .
$$


a)

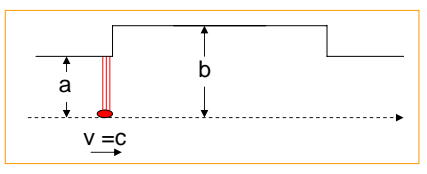

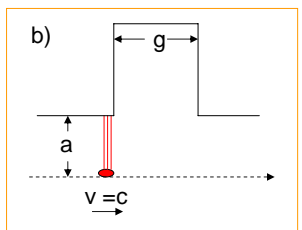

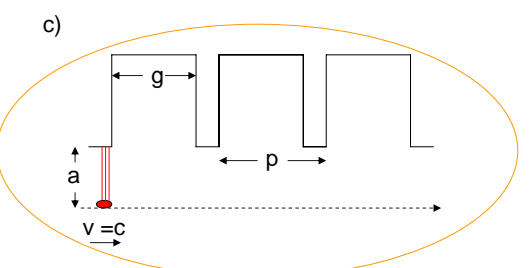

Figure 3. Structure geometry for diffraction wakes: (a) shallow transition pair, (b) deep cavity, (c) periodic array of deep cavities. The on-axis bunch and its electric field are indicated in red.

For a deep cavity with beam pipes (see Fig. 3b) the high frequency impedance, $Z \propto \sqrt{g / k} / a$, and the wake ${ }^{3,11}$

$$
W(s)=\frac{Z_{0} c}{\sqrt{2} \pi^{2} a} \sqrt{\frac{g}{s}} .
$$

For the transverse case $Z_{x} \propto g^{1 / 2}(k a)^{-3 / 2}$, and

$$
W_{x}(s)=\frac{2^{3 / 2} Z_{0} c}{\pi^{2} a^{3}} \sqrt{g s}
$$

the longitudinal and dipole cases are related according to Eqs. 3. Note that a cavity is considered shallow (deep) to a bunch of total length $\ell$ if $g \gg(\ll)\left[2(b-a)^{2} / \ell\right]$. This is a statement that radiation, generated by the head of the bunch as it enters the cavity, can (cannot) reflect off the outer wall and return in time to meet the bunch tail.

In accelerators with longer bunches, tapering of transitions is often used as a way of reducing their wakefield effect. For short bunches, however, radiation is diffracted forward at a shallow angle, $\theta \sim \sigma_{z} / a$, and until the taper angle becomes comparable to this quantity the tapering has little effect. In the undulator region of the LCLS there are transitions, flanges, etc, objects that generate diffraction wakes; however, with $\sigma_{z}=20 \mu \mathrm{m}$ and $a=2.5 \mathrm{~mm}$, tapering would have no effect unless $\theta \lesssim 0.5^{\circ}$.

In a first step in generating an impedance budget for a project it is sometimes useful to calculate the first and second moments of the bunch 
wake for the various impedance objects; these are then compared to estimate the relative importance of the different objects for-energy loss, energy spread, transverse wake, etc. In Table 1, the first and second moments of wake forms for Gaussian bunches, including those for diffraction wakes and other wakes discussed later in this report, are given.

Table 1. First and second moments of (Gaussian) bunch wakes, $\left\langle\mathcal{W}_{g}\right\rangle$ and $\left(\mathcal{W}_{g}\right)_{r m s}$, corresponding to common short-bunch wake forms $W(s)$. Here the $s$ dependence alone is considered. $H(s)$ is the unit step function ( $=0$ for $s<0 ; 1$ for $s>0$ ).

\begin{tabular}{llcc}
\hline Wake form, $W(s)$ & Wake example & $\left\langle\mathcal{W}_{g}\right\rangle$ & $\left(\mathcal{W}_{g}\right)_{r m s}$ \\
\hline Circuit Models: & & $\frac{1}{2 \sqrt{\pi} \sigma_{z}}$ & $\frac{0.111}{\sigma_{z}}$ \\
Resistive: $\delta(s)$ & Shallow transition pair, $W$ & $\frac{1}{2}$ & $\frac{1}{\sqrt{3}}$ \\
Capacitive: $H(s)$ & Shallow transition pair, $W_{x}$ & 0 & $\frac{1}{\sqrt{6 \pi} 3^{1 / 4} \sigma_{z}^{2}}$ \\
Inductive: $\delta^{\prime}(s)$ & Roughness, inductive model, $W$ & & \\
\hline Power Law, $s^{\alpha}:$ & & $\frac{0.723}{\sqrt{\sigma_{z}}}$ & $\frac{0.292}{\sqrt{\sigma_{z}}}$ \\
$\alpha=-\frac{1}{2}$ & Deep cavity, $W$ & $0.489 \sqrt{\sigma_{z}}$ & $0.374 \sqrt{\sigma_{z}}$ \\
$\alpha=\frac{1}{2}$ & Deep cavity, $W x$ & $\frac{-0.489}{\sigma_{z}^{3 / 2}}$ & $\frac{0.516}{\sigma_{z}^{3 / 2}}$ \\
$\alpha=-\frac{3}{2}$ & Resistive wall, low freq., $W$ & $\frac{-0.758}{\sigma_{z}^{4 / 3}}$ & $\frac{0.532}{\sigma_{z}^{4 / 3}}$ \\
$\alpha=-\frac{4}{3}$ & CSR, $W$ &
\end{tabular}

In the undulator region of the LCLS there are 30 rectangular-to-round transition pairs. A 10 by $5 \mathrm{~mm}$ rectangular cross-section becomes a round pipe with $a=4 \mathrm{~mm}$ in the BPM's. A pessimistic calculation considers a cylindrically symmetric transition pair with $a=2.5 \mathrm{~mm}$ and $b=4 \mathrm{~mm}$ and uses Eq. 6 and the "Resistive" entry in Table 1. The rms induced energy spread, for all transition pairs, is then estimated to be $\left(E=14 \mathrm{GeV}, \sigma_{z}=\right.$ $20 \mu \mathrm{m}): \Delta E_{r m s} / E=0.02 \%$. A full 3D simulation of the real geometry also finds a resistive wake, but with $\Delta E_{r m s} / E=0.013 \%{ }^{12}$. In the undulator region of the LCLS, the wake effect of the transitions is small compared to that of the resistive wall wake (discussed later), and can be ignored. 


\subsubsection{Periodic Structure}

For an array of cavities with period $p$ (see Fig. 3c), the asymptotic, high frequency impedance is given by ${ }^{4,13}$,

$$
Z(k) \approx \frac{i Z_{0}}{\pi k a^{2}}\left[1+(1+i) \frac{\alpha(g / p) p}{a}\left(\frac{\pi}{k g}\right)^{1 / 2}\right]^{-1},
$$

with $\alpha(x) \approx 1-0.465 \sqrt{x}-0.070 x$; the real part of the impedance $\operatorname{Re}(Z) \sim$ $k^{-3 / 2}$. Inverse Fourier transforming, one obtains an analytical expression for the very short-range wake:

$$
W(s) \approx \frac{Z_{0} c}{\pi a^{2}} \exp \left(\frac{2 \pi \alpha^{2} p^{2} s}{a^{2} g}\right) \operatorname{erfc}\left(\frac{\alpha p}{a} \sqrt{\frac{2 \pi s}{g}}\right),
$$

with erfc the compliment error function.

Obtaining the short-range wake numerically and fitting to a simple function, we obtain a result that is valid over a larger $s$ range and over a useful range of structure parameters ${ }^{14}$ :

$$
W(s)=\frac{Z_{0} c}{\pi a^{2}} \exp \left(-\sqrt{s / s_{1}}\right),
$$

with

$$
s_{1}=0.41 \frac{a^{1.8} g^{1.6}}{p^{2.4}} .
$$

The result is valid for $s / p \leq 0.15,0.34 \leq a / p \leq 0.69$, and $0.54 \leq g / p \leq$ 0.89 . For the SLAC linac $(a=11.6 \mathrm{~mm}, g=29.2 \mathrm{~mm}, p=35.0 \mathrm{~mm})$ $s_{1}=1.5 \mathrm{~mm}$. It has been numerically verified that, for a Gaussian bunch in a periodic accelerator structure, the steady state result becomes valid after a distance of $z \approx a^{2} / 2 \sigma_{z}{ }^{15}$. For Linac-3 of the LCLS where $\sigma_{z}=20 \mu \mathrm{m}$, the distance to steady-state is $3.4 \mathrm{~m}$ if the bunch were Gaussian, is $\sim 35 \mathrm{~m}$ for the actual double horned distribution; but even this distance is small compared to the $550 \mathrm{~m}$ length of linac, and the transient region can be ignored.

The high frequency transverse impedance can be shown to be related to the high frequency longitudinal impedance (Eq. 10) according to $Z_{x}=$ $2 Z / k a^{2}{ }^{16}$. As with the longitudinal case, the short-range dipole wake was obtained numerically for a set of parameters and then fit to a simple function ${ }^{17}$ :

$$
W_{x}(s)=\frac{4 Z_{0} c s_{2}}{\pi a^{4}}\left[1-\left(1+\sqrt{\frac{s}{s_{2}}}\right) \exp \left(-\sqrt{\frac{s}{s_{2}}}\right)\right],
$$


with $s_{2}=0.17 a^{1.79} g^{0.38} / p^{1.17}$. This equation is valid over the same parameter regime as in the longitudinal case.

Real linear accelerator structures are often not perfectly periodic; socalled "constant gradient" (e.g. the SLAC linac) or "detuned" structures have gradual variations in cell geometry along the structure. However, for the short-range wake/high frequency (broad-band) impedance of such structures the equations given here still apply (with possible slight adjustments of parameters, where e.g. $a$ is replaced by effective iris radius, etc). The point charge wakes and (Gaussian) bunch wakes in the SLAC linac are plotted in Fig. 4.
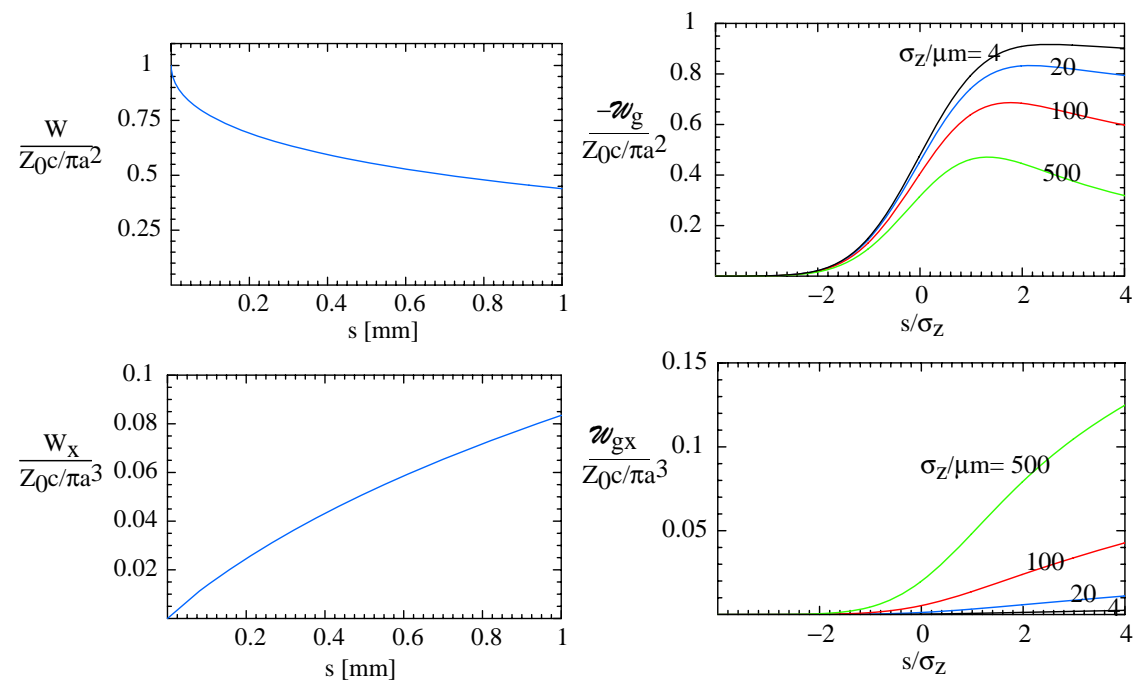

Figure 4. For the SLAC linac: the point charge wakes, $W$ and $W_{x}$ (left plots), and the bunch wakes for Gaussian bunches, $\mathcal{W}_{g}$ and $\mathcal{W}_{g x}$, for various bunch lengths (right plots). The scaling factors $Z_{0} c / \pi a^{2}=0.27 \mathrm{GV} /(\mathrm{nC}-\mathrm{km})$ and $Z_{0} c / \pi a^{3}=23 \mathrm{MV} /(\mathrm{nC}-\mathrm{mm}-\mathrm{km})$.

In the LCLS the longitudinal wakes induced in the linacs are integral parts of the longitudinal phase space manipulation process and thus need to be known accurately. The LCLS beam line has been designed with the aid of a macro-particle, longitudinal phase space tracking program, LiTrack ${ }^{18}$, using the function $W(s)$ for the SLAC linac as an input. Measurements at SLAC confirmed such calculations down to $\sigma_{z} \sim 0.5 \mathrm{~mm}$ many years ago ${ }^{19}$, and down to $\sigma_{z} \sim 50 \mu \mathrm{m}$ in more recent times ${ }^{20,21}$.

In Linac- 1 and 2 of the LCLS the sum of the wake and the applied 
$\mathrm{RF}$ wave generates the energy/longitudinal position correlation needed for bunch compression. For example, in Linac-2 the bunch phase offset is $\sim-40^{\circ}$ (ahead of the $\mathrm{rf}$ crest), the rms bunch shape is roughly Gaussian with $\sigma_{z}=190 \mu \mathrm{m}$, and the total structure length is $330 \mathrm{~m}$. The energy/position correlation induced by the $\mathrm{RF}$ is $\sim 250 \mathrm{MeV} / \mathrm{mm}$, by the wake $\sim-100 \mathrm{MeV} / \mathrm{mm}$. In Linac-3, which is $550 \mathrm{~m} \mathrm{long,} \mathrm{where} \mathrm{the} \mathrm{bunch}$ shape is double horned with $\mathrm{rms} 20 \mu \mathrm{m}$, the wake cancels a residual correlation of $850 \mathrm{MeV} / \mathrm{mm}$, left at the end of BC-2. Finally, note that it is due the non-linear, cubic behavior in $\mathcal{W}(s)$ in Linac-2 that the final LCLS bunch shape contains the unavoidable horns.

\subsection{Roughness Impedance}

A metallic beam pipe with a rough surface has an impedance that is enhanced at high frequencies. Two approaches to modeling the impedance of a rough surface are with: (i) a random collection of bumps on a surface and (ii) a wall with small periodic corrugations.

An early model of roughness impedance assumes a random, noninteracting collection of bumps of various shapes (see sketch Fig. 5), with the total impedance given by the sum of the individual impedances. Consider a beam pipe of radius $a$ on which there is a small hemispherical bump of radius $h$. At low frequencies $k \ll 1 / h$ the impedance is inductive and given by ${ }^{22}$

$$
Z(k)=i k c \mathcal{L}_{1}=i k \frac{Z_{0} h^{3}}{4 \pi a^{2}},
$$

with $\mathcal{L}_{1}$ the inductance of the bump. If the hemispherical bump is replaced by one of a different shape but of about the same size, the above equation is multiplied by a form factor of order 1 . For many randomly distributed bumps the inductance per unit length $\mathcal{L} / L$ can be written as ${ }^{23}$

$$
\mathcal{L} / L=\frac{2 \alpha f a \mathcal{L}_{1}}{h^{2}}=\frac{\alpha f Z_{0} h}{2 \pi a c},
$$

where $\alpha$ is the filling factor of bumps and $f$ is the effective form factor. The rms wake of a Gaussian bunch is given by $\mathcal{W}_{r m s} \approx 0.06 c^{2} \mathcal{L} / L \sigma_{z}^{2}$.

The simple idea of this model has been systematized so that one can, from measurements of the contour of a surface, obtain the inductance per length of the surface ${ }^{24}$

$$
\mathcal{L} / L=\frac{Z_{0}}{2 \pi c a} \int_{-\infty}^{\infty} \frac{k_{z}^{2}}{\sqrt{k_{\theta}^{2}+k_{z}^{2}}} S\left(k_{z}, k_{\theta}\right) d k_{z} d k_{\theta},
$$




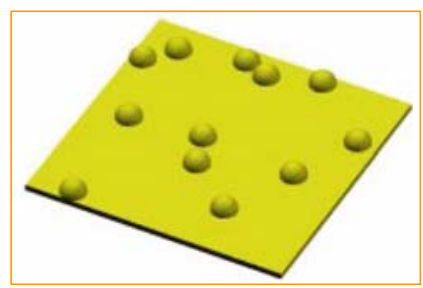

Figure 5. Random collection of bumps model of roughness impedance.

where $S\left(k_{z}, k_{\theta}\right)$ is the spectrum of the surface profile - the square of the absolute value of the Fourier transform of the surface variation-and $k_{z}$, $k_{\theta}$, are the wave numbers in the longitudinal and azimuthal directions. Finally, note that since, for an inductive model, the wake of a bunch is given by the derivative of the bunch distribution, such a model cannot be applied to a rectangular or other non-smooth bunch shape.

The second modeling approach is to consider a beam pipe with small periodic corrugations (see Fig. 6). The motivation for such a model came from numerical simulations of many randomly placed, small cavities on a beam pipe; it was found that, in steady state, the short range wake is very similar to the truly periodic case ${ }^{5}$. Consider a beam pipe with small, rectangular, periodic corrugations, with $h$ the half-depth, $g$ the gap $(=p / 2)$, and $p$ the period. In the case $h / p \gtrsim 1$ the wake is dominated by one mode of relatively low frequency $\left(k_{0} \ll 1 / h\right)^{5,6}$ :

$$
W(s) \approx \frac{Z_{0} c}{\pi a^{2}} \cos k_{0} s \quad \text { with } \quad k_{0}=\frac{2}{\sqrt{a h}} .
$$

For smooth bunches, with $k_{0} \sigma_{z}$ large, the wake for this model becomes inductive with $\mathcal{L} / L=Z_{0} h / 4 a c$, very similar to the first model. However, even for non-smooth distributions this model can be applied. Note that the dipole wake for this model is also given by a single mode at the same frequency.

This single resonator model is valid when the depth-to-period of the surface roughness is not small compared to 1 . However, measurements of copper surfaces with good finish show that the opposite tends to be true: that the depth-to-period $\lesssim 0.01$ (see Fig. 7$)^{25}$. As the depth-to-period ratio becomes small the dominant, low frequency mode is replaced by many weak, closely spaced modes beginning just above $k=\pi / p^{26}$. For a sinusoidally oscillating wall with amplitude $h$ and period $p$, where $h \ll p$, the wake was 


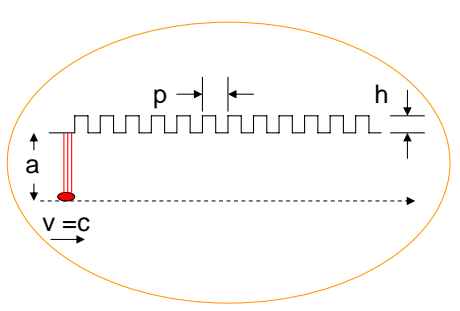

Figure 6. Geometry for small corrugations model of roughness impedance.

found by Stupakov to be ${ }^{27}$ :

$$
\begin{gathered}
W(s)=\frac{Z_{0} c h^{2} k_{1}^{3}}{4 \pi a} f\left(k_{1} s\right), \\
f(\zeta)=\frac{1}{2 \sqrt{\pi}} \frac{\partial}{\partial \zeta} \frac{\cos (\zeta / 2)+\sin (\zeta / 2)}{\sqrt{\zeta}},
\end{gathered}
$$

with $k_{1}=2 \pi / p$ (the function $f(\zeta)$ is shown in Fig. 8a). The formula is valid for $s \gtrsim k_{1} h^{4 / 3} a^{2 / 3}$. For bunch length $\sigma_{z} \lesssim k_{1}^{-1}$ all we need is the (small $s$ ) leading order term:

$$
W(s)=-\frac{Z_{0} c h^{2}}{16 \pi^{3 / 2} a}\left(\frac{k_{1}}{s}\right)^{3 / 2} .
$$

In this case the wake of a Gaussian bunch

$$
\mathcal{W}_{g}(s)=W\left(\sigma_{z}\right) w_{g}\left(s / \sigma_{z}\right)
$$

where the form factor $w_{g}$ is shown in Fig. 8b. Note that for given $h$ the bunch wake here is weaker, by $\sim h / p$, than for the single mode model described earlier. Note also that the wake has the form of the long-range resistive wall wake; it can be described as the wake of a metal with conductivity $\sigma=16 /\left(Z_{0} h^{4} k_{1}^{3}\right)$.

In the undulator region of the LCLS, $\sigma_{z}=20 \mu \mathrm{m}$ and $a=2.5 \mathrm{~mm}$. If we believe Fig. 7 is representative of the undulator beam pipe surface roughness $(h \sim 0.5 \mu \mathrm{m}, p \sim 100 \mu \mathrm{m})$ then our parameters are in the regime of Stupakov's model. In this case the roughness wake is small, only $\sim 0.15$ the strength of the resistive wall wake (discussed below).

\subsection{CSR Wake}

The effect on a bunch of coherent synchrotron radiation can be described in terms of a wakefield, although there are some differences from normal 


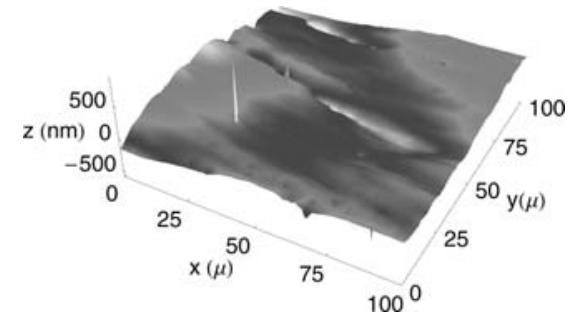

Figure 7. A sample surface profile measured with an atomic force microscope (from Ref. [25]).
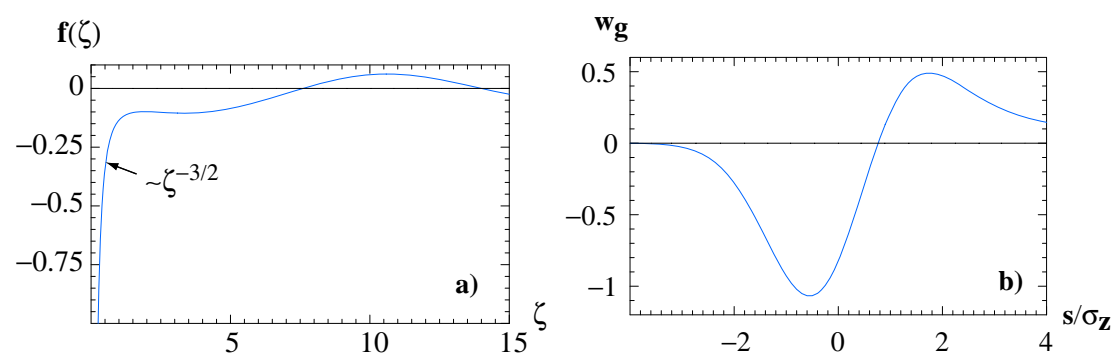

Figure 8. The wake of shallow, periodic corrugations $(h \ll p \ll a)$ : form factor for wake a), for Gaussian bunch wake $\left[k_{1} \sigma_{z} \lesssim 1\right] \mathrm{b}$ ).

wakes. For example, for an ultra-relativistic particle moving in a circle of radius $R$ in free space (see Fig. 9), the wake (for a test particle on the same path) is non-zero ahead of the radiating particle $(s<0)$, because the radiation moves on a shorter, straight path. The wake experienced by the test particle is sketched in Fig. 10a. For $(-s) \gg R / \gamma^{3}$ it is given by ${ }^{28,29}$

$$
W(s)=-\frac{Z_{0} c}{2 \cdot 3^{4 / 3} \pi R^{2 / 3}(-s)^{4 / 3}} \quad s<0,
$$

while $W\left(0^{-}\right)=Z_{0} c \gamma^{4} / 3 \pi R^{2}$. The wake of a Gaussian bunch ${ }^{28,31}$

$$
\mathcal{W}_{g}(s)=W\left(-\sigma_{z}\right) w_{g}\left(s / \sigma_{z}\right)
$$

where the form factor $w_{g}\left(s / \sigma_{z}\right)$ is plotted in Fig. 10b. The impedance, the Fourier transform of the wake, is ${ }^{28,29,30}$

$$
Z(k)=\frac{Z_{0}}{2 \cdot 3^{1 / 3} \pi} \Gamma\left(\frac{2}{3}\right) e^{i \pi / 6} \frac{k^{1 / 3}}{R^{2 / 3}},
$$

with $\Gamma(2 / 3)=1.35$, valid to very high frequencies $\left(k \sim \gamma^{3} / R\right)$.

For particles moving in a circle through a beam pipe the wake will be modified, an effect that can be calculated using image charges in the time 


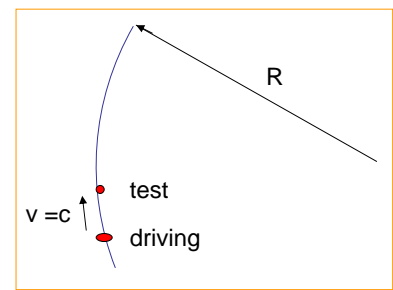

Figure 9. Geometry for CSR wake calculation: the driving and test particles follow a circular path of radius $R$ in free space.
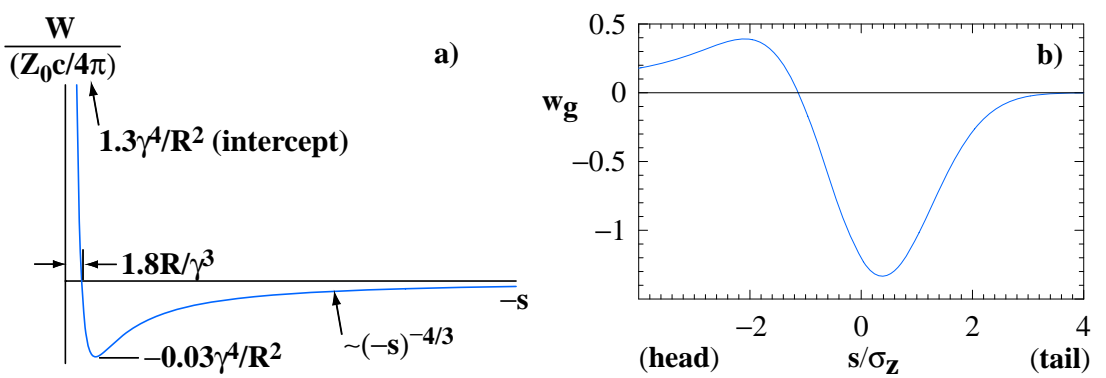

Figure 10. a) Sketch of the CSR wake of a particle, in free space, moving on a circle of radius $R$; b) bunch wake form factor for a Gaussian bunch.

domain ${ }^{28}$, or be dealt with in the frequency domain ${ }^{31}$. The pipe suppresses the wake of a bunch, provided that $\sigma_{z} / a \gtrsim(a / R)^{1 / 2} 31$. If we consider the beam in the last bend of BC2 of the LCLS, and take $\sigma_{z}=20 \mu \mathrm{m}, a=1 \mathrm{~cm}$ (here the half the vertical beam pipe aperture), and $R=15 \mathrm{~m}$, we see that the bunch length is 13 times too short to feel the effect of shielding.

Chicanes are composed of 3 or 4 bends with drifts in between. Instead of one continuous circle, the beam moves in circular arcs (in the bends) and straight lines (outside the bends). Analytical solutions for the 1D wake of an ultra-relativistic particle entering, traversing, and leaving a bend without shielding have been derived ${ }^{32}$. There are transients when the beam enters a bend and also after it leaves. The catch-up distance is the distance a test particle, ahead of the radiating particle, needs to travel to catch up to the radiated wave. The distance of the incoming transient region is $z \approx\left(24 R^{2} \sigma_{z}\right)^{1 / 3}$ (assuming $z / R$ is small) ${ }^{29}$.

One can consider the potential energy change (the "compression work") that the beam undergoes in being compressed. The average kinetic energy change to balance this is approximately (assuming the compression factor 
is large, for a Gaussian bunch) ${ }^{33,34}$

$$
\langle\Delta E\rangle=-\frac{e N Z_{0} c}{4 \pi^{3 / 2} \sigma_{z}} \ln \left(\frac{\gamma \sigma_{z}}{\sigma_{x}+\sigma_{y}}\right)
$$

where beam sizes are final quantities; the rms spread $\Delta E_{r m s} \approx-0.4\langle\Delta E\rangle$. Interestingly, for example chicanes, Eq. 26 was found to roughly agree with the total energy change obtained by detailed CSR simulations ${ }^{34}$.

To simulate the CSR force in a chicane compressor, computer programs that slice the bunch into macro-particles and solve the Liénard-Wiechert equations have been written ${ }^{35,36,37}$, as has one that employs a frequency domain method ${ }^{38}$. In these programs the bunch can have transverse as well as longitudinal dimensions, shielding can be added, and the orbit/forces can be computed in a self-consistent manner. These programs, however, can be time consuming to run. The 1D analytical wake formulas for a particle entering, traversing, and leaving a bend, when used in a tracking program such as $E L E G A N T^{39}$, give results that are quick to obtain and agree reasonably well with more detailed simulations for typical beam/chicane parameters 37 . Reference ${ }^{37}$ gives a comparison of some simulation programs.

CSR induced energy spread in a chicane, through the dispersion, leads to emittance growth. For the BC-2 chicane of the LCLS, we can estimate the rms energy spread increase by taking 0.4 times the Eq. 26 result (a Gaussian approximation, with $\left.E=4.5 \mathrm{GeV}, \sigma_{z}=20 \mu \mathrm{m}, \sigma_{x}=\sigma_{y}=25 \mu \mathrm{m}\right)$, yielding $\Delta E_{r m s} / E=0.018 \%$. ELEGANT simulation gives $0.016 \%$ (good

agreement), and a resulting $x$ emittance growth of $38 \%{ }^{40}$. Finally, note that CSR has been a much studied topic in recent years; this has only been a cursory view of the subject.

\subsection{Resistive Wall Wake}

The impedance of a round metallic beam pipe of radius $a$ has long been known to be ${ }^{3}$

$$
Z(k)=\left(\frac{Z_{0}}{2 \pi a}\right)\left[\frac{\lambda}{k}-\frac{i k a}{2}\right]^{-1}
$$

where parameter $\lambda$ is given by

$$
\lambda=\sqrt{\frac{Z_{0} \sigma|k|}{2}}[i+\operatorname{sign}(\mathrm{k})],
$$


with $\sigma$ the (dc) conductivity of the metal. Inverse Fourier transforming the impedance, one obtains the wake ${ }^{3,41}$

$$
W(s)=\frac{4 Z_{0} c}{\pi a^{2}}\left(\frac{e^{-s / s_{0}}}{3} \cos \frac{\sqrt{3} s}{s_{0}}-\frac{\sqrt{2}}{\pi} \int_{0}^{\infty} \frac{d x x^{2} e^{-x^{2} s / s_{0}}}{x^{6}+8}\right),
$$

where the characteristic distance $s_{0}$ is given by

$$
s_{0}=\left(\frac{2 a^{2}}{Z_{0} \sigma}\right)^{\frac{1}{3}}
$$

(see Fig. 11). The long-range asymptote, corresponding to the low frequency part of the impedance, is given by ${ }^{3}$

$$
W(s)=-\frac{c}{4 \pi^{3 / 2} a} \sqrt{\frac{Z_{0}}{\sigma}} \frac{1}{s^{3 / 2}},
$$

a formula that is valid provided that $s \gg s_{0}$. Note that, unlike the long range wake, the general wake formula does not scale as $\sigma^{-1 / 2}$. The transverse wake can be obtained from the longitudinal wake using Eq. 3.

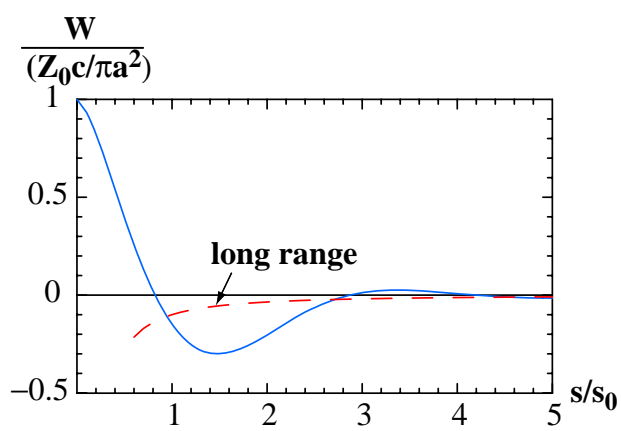

Figure 11. Plot of Eq. 29, the resistive wall wake. The long-range asymptote (Eq. 31) is shown in dashes.

For the LCLS in the undulator region, the rms bunch length $\sigma_{z}=20 \mu \mathrm{m}$, and $a=2.5 \mathrm{~mm}$; if the beam pipe is made of copper $\left(\sigma=6.5 \times 10^{7} \Omega^{-1} \mathrm{~m}^{-1}\right)$ then $s_{0}=8.0 \mu \mathrm{m}$; if it is made of aluminum $\left(\sigma=4.2 \times 10^{7} \Omega^{-1} \mathrm{~m}^{-1}\right)$, $s_{0}=9.2 \mu \mathrm{m}$. Thus we see that $\sigma_{z} \ngtr s_{0}$. In addition, the bunch in the undulator region is not Gaussian; it is a double-horned distribution with very high frequency content $\left(k \sim[0.05,0.5] \mu \mathrm{m}^{-1}\right.$, whose upper limit $\left.\gg 1 / s_{0}\right)$. Thus the long-range wake will not suffice, and the general solution is needed. 
The problem with using Eq. 29 for the short bunch wake is that it assumes that the conductivity of the metal wall is constant, i.e. independent of frequency. It is believed, however, that electrical conductivity in normal metals, up to the highest frequencies of interest to us $\left(k \sim 0.5 \mu \mathrm{m}^{-1}\right)$, is described by the Drude-Sommerfeld free-electron model of conductivity 42,43 . According to this model the dc conductivity of a metal is given by $\sigma=n e^{2} \tau / m$, with $n$ the density of conduction electrons; with $e$ the charge, $\tau$ the relaxation time, and $m$ the mass of the electron. In this model the ac conductivity, a response to applied oscillating fields, is given by

$$
\tilde{\sigma}=\frac{\sigma}{1-i k c \tau} \text {. }
$$

The dc wake equation, Eq. 29, is valid provided $\Gamma=c \tau / s_{0} \ll 1$. For copper at room temperature $c \tau=8.1 \mu \mathrm{m}$, for aluminum $c \tau=2.4 \mu \mathrm{m}$; if $a=2.5 \mathrm{~mm}: \Gamma=1.0(\mathrm{Cu}), \Gamma=0.26(\mathrm{Al})$. We see that the dc wake equation is not valid for LCLS undulator beam pipe properties.

The calculation of the resistive wall wake including ac conductivity again involves (numerically) inverse Fourier transforming the impedance (Eq. 27), but with $\sigma$ in parameter $\lambda$ (Eq. 28) replaced by $\tilde{\sigma}^{41}$. For the special case $\Gamma \gtrsim 1$ (and $s$ not too large) the wake can be approximated as a damped resonator

$$
W(s) \approx \frac{Z_{0} c}{\pi a^{2}} e^{-s / c \tau} \cos \left[\sqrt{2 k_{p} / a}\right] \quad[\Gamma \gtrsim 1],
$$

with $k_{p}=\sqrt{Z_{0} \sigma / c \tau}$, the plasma frequency of the metal. This approximation can e.g. be used for the LCLS undulator beam pipe if it is copper. In Fig. 12 we plot the ac wakes for the LCLS undulator beam pipe, for both copper and aluminum. Note that the ac wake of aluminum damps more rapidly than that of copper, due to its smaller value of $\tau$. The dc wake for copper is also shown, for comparison (remember, this result is not in accord with the free electron model and thus non-physical).

The energy change, $\Delta E(s)$, induced by the resistive wall wake in the undulator region of the LCLS, for cases of copper and aluminum beam pipes, is shown in Fig. 13 ( $\Delta E>0$ indicates energy gain). Here energy $E=14 \mathrm{GeV}$, pipe length $L=130 \mathrm{~m}$, and the beam shape is double horned with rms length $20 \mu \mathrm{m}$ (the bunch shape is superimposed by the dashed curve in the figure). The sharp rise of the leading horn in the bunch shape results in wakes that ring; the ringing amplitude is about twice as large for copper than aluminum, due to the difference in relaxation times in the two metals. Note that other wakefield contributors in the undulator region, 


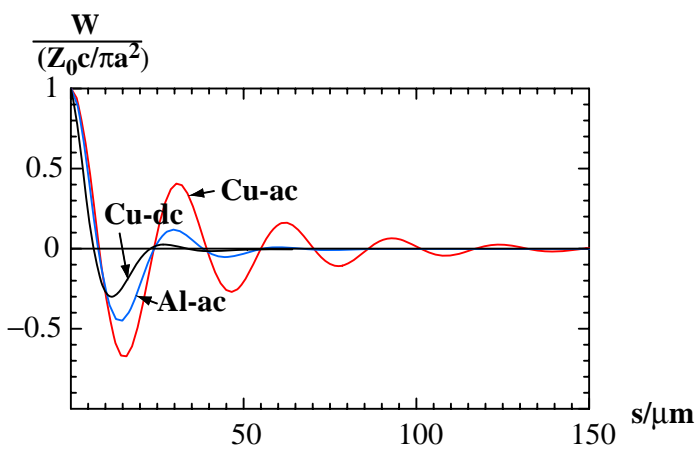

Figure 12. The dc and ac wakes for copper, and the ac wake for aluminum, for the LCLS undulator beam pipe $(a=2.5 \mathrm{~mm})$.

such as wall roughness, transitions, and BPM's, are weak in comparison to the resistive wall, and can be ignored. From FEL simulations for LCLS parameters, it appears that particles within a window $\Delta E / E \lesssim 0.3 \%$ will lase ${ }^{44}$. Because of uncertainties in the emittance and energy spread within the horns of the beam, it is not clear what parts of these will lase. As for the region of beam between the horns, for copper the energy deviation is large compared to $0.3 \%$, for aluminum it is smaller. It appears, according to FEL simulations, that more of the beam between the horns will lase in the case of aluminum than in the case of copper ${ }^{44}$.

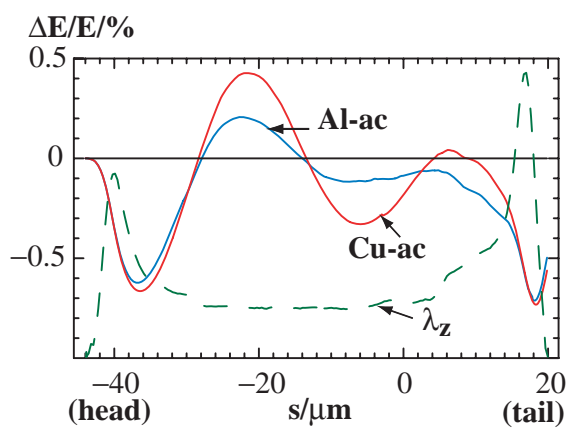

Figure 13. For LCLS parameters: the total energy variation induced within the $1 \mathrm{nC}$ bunch in the $130 \mathrm{~m}$ long LCLS undulator beam pipe. Results are given for round beam pipes of copper and aluminum. Superimposed is the bunch shape, with the head to the left (the dashes).

The resistive wall wake of a flat beam pipe (rectangular with width 
much greater than height) has also been computed ${ }^{45}$. Keeping the vertical aperture of the LCLS undulator beam pipe fixed, the wake effect can be reduced by about $30 \%$ by going to a flat geometry (this is the chosen LCLS beam pipe shape, aluminum is the chosen surface material). Another effect that can be noticeable at high frequencies is the (room temperature) anomalous skin effect; here it appears to be a $\sim 15 \%$ effect ${ }^{45}$. Finally, one may ask the question, What confidence do we have that the free-electron model is valid in the frequency range of interest to us, namely $k$ in the range $[0.05,0.5] \mu \mathrm{m}^{-1}$ ? Reflectivity measurements can be used to study this question. However, over our frequency range of interest, such measurements are difficult to do with sufficient accuracy, since the reflectivity of metals becomes very nearly 1 ; little data over this range is found in the literature. Recently, however, reflectivity measurements were performed at the synchrotron light source at Brookhaven on $\mathrm{Cu}$ and $\mathrm{Al}$ samples; the results are in reasonably good agreement with our assumed free-electron model ${ }^{46}$.

\section{Conclusion}

We have discussed wakefields excited by short bunches in accelerators. In particular, we have reviewed some of what has been learned in the last 10-15 years concerning diffraction wakes, roughness impedance, coherent synchrotron radiation wakes, and the resistive wall wake. We have studied the importance of these wakes in the Linac Coherent Light Source (LCLS) project. The longitudinal accelerator structure wake in the SLAC linac is an important ingredient in the bunch compression process. Of the wakes in the undulator region of the LCLS, the dominant one is the resistive wall wake. To weaken its effect, the undulator beam pipe was chosen to be aluminum instead of copper, and of flat geometry instead of round.

\section{Acknowledgements}

The author thanks G. Stupakov for discussions about short-range wakes, P. Emma for discussions on the LCLS and its phase space manipulation. In addition, he thanks P. Emma for Fig. 1, A. Novokhatski for Fig. 2, and G. Stupakov for Figs. 5 and 7.

\section{References}

1. "LCLS Design Report," SLAC-R 593, 2002. 
2. S. Chattopadhyay, editor, Part. Accel. 25, 1990.

3. A. Chao, Physics of Collective Beam Instabilities in High Energy Accelerators (Wiley \& Sons, New York, 1993).

4. R. Gluckstern, Phys. Rev. D39, 2780 (1989).

5. A. Novokhatski and A. Mosnier, in Proceedings of the Particle Accelerator Conference, Vancouver, Canada, 1997 (IEEE, Piscataway, NJ, 1997), p. 1661 .

6. K. Bane and A. Novokhatski, SLAC-AP 117, March 1999.

7. K.-Y. Ng, Phys. Rev. D42, 1819 (1990).

8. H. Henke and O. Napoli, in Proceedings of the European Particle Accelerator Conference, Nice, France, 1990 (Editions Frontieres, Gif-sur-Yvette, France, 1991), p. 1046.

9. S. Heifets and S. Kheifets, Rev. Mod. Phys. 63, 631 (1991).

10. E. Gianfelice and L. Palumbo, IEEE Trans. Nucl. Sci. 32, 2, 1084 (1990).

11. K. Bane and M. Sands, Part. Accel. 25, 73 (1990).

12. K. Bane and I. Zagorodnov, SLAC-PUB-11388, August 2005.

13. K. Yokoya and K. Bane, in Proceedings of the Particle Accelerator Conference, New York, NY, 1999 (IEEE, Piscataway, NJ, 1999), p. 1725.

14. K. Bane, A. Mosnier, A. Novokhatski, K. Yokoya, in Proceedings of the International Computational Accelerator Physics Conference, Monterey, CA, 1998 (SLAC, 1998) SLAC-R-580, p. 137.

15. K. Bane, M. Timm, T. Weiland, in Proceedings of the Particle Accelerator Conference, Vancouver, Canada, 1997 (IEEE, Piscataway, NJ, 1997), p. 515.

16. A. Fedetov, R. Gluckstern, M. Venturini, Phys. Rev. ST-AB 2, 064401 (1999).

17. K. Bane, SLAC-PUB-9663, March 2003.

18. K. Bane and P. Emma, in Proceedings of the Particle Accelerator Conference, Knoxville, TN, 2005 (IEEE, Piscataway, NJ, 2005), p. 4266.

19. K. Bane, F.-J. Decker, J. Seeman, F. Zimmermann, in Proceedings of the Particle Accelerator Conference, Vancouver, Canada, 1997 (IEEE, Piscataway, NJ, 1997), p. 1876.

20. K. Bane et al, in Proceedings of the Particle Accelerator Conference, Portland, OR, 2003 (IEEE, Piscataway, NJ, 2003), p. 3126.

21. C. Barnes, Stanford University, PhD Thesis, SLAC-R-799, March 2006.

22. S. Kurennoy, Phys. Rev. E55, 3529 (1997).

23. K. Bane, C.K. Ng, A.W. Chao, in Proceedings of the Particle Accelerator Conference, Vancouver, Canada, 1997 (IEEE, Piscataway, NJ, 1997), p. 1738.

24. G. Stupakov, Phys. Rev. ST-AB 1, 064401 (1998).

25. G. Stupakov, R. Thomson, D. Waltz, R. Carr, Phys. Rev. ST-AB 2, 060701 (1999).

26. M. Chatard-Moulin and A. Papiernik, in Proceedings of the Particle Accelerator Conference, San Francisco, CA, 1979; published as IEEE Trans. Nucl. Sci. NS-26, 3, p. 3523.

27. G. Stupakov, in Proceedings of the Physics of, and Science with, the X-Ray Free-Electron Laser, Arcidosso, Italy, 2000 (Amer. Inst. Phys., Melville, NY, 2000), p. 141. 
28. J.B. Murphy, S. Krinsky, R. Gluckstern, Part. Accel. 57, 9 (1997), and in Proceedings of the Particle Accelerator Conference, Dallas, TX, 1995 (IEEE, Piscataway, NJ, 1996), p. 2980.

29. Ya. S. Derbenev, J. Rossbach, E. L. Saldin, V. D. Shiltsev, DESY-TESLAFEL 95-05, DESY, 1995.

30. R. L. Warnock and P. Morton, SLAC-PUB-4562, March 1998, and Part. Accel. 25, 113 (1990).

31. R. Warnock, in Proceedings of 4th Advanced ICFA Beam Dynamics Workshop, Tsukuba, Japan, 1990 (KEK, Tsukuba, Japan, 1990), p. 151, and in Proceedings of the Particle Accelerator Conference, San Francisco, CA, 1991 (IEEE, Piscataway, NJ, 1991), p. 1824.

32. E. Saldin, et al, Nucl. Inst. Meth. A398, 373 (1997).

33. M. Dohlus, talk given at CSR-Workshop-2002 at Zeuten, Germany; see http://www.desy.de/csr/csr_workshop_2002.

34. K. Bane and A. Chao, Phys. Rev. ST-AB 5, 104401 (2002).

35. A. Kabel, et al, Nucl. Inst. Meth. A455, 180 (2000).

36. R. Li, Nucl. Inst. Meth. A429, 310 (1998).

37. L. Giannessi, Phys. Rev. ST-AB 6, 114802 (2003).

38. T. Agoh and K. Yokoya, Phys. Rev. ST-AB 7, 054403 (2004).

39. M. Borland, Phys. Rev. ST-AB 4, 070701 (2001).

40. P. Emma, private communication.

41. K. Bane and M. Sands, in Proceedings of Micro Bunches Workshop Upton, NY, 1995 (Amer. Inst. Phys., Woodbury, NY, 1995), p. 131.

42. N. Ashcroft and N. Mermin, Solid State Physics, (Harcourt Brace Publishers, Orlando, FL, 1976).

43. E. Shiles et al, Phys. Rev. B4, 1612 (1980).

44. Z. Huang and G. Stupakov, in Proceedings of the Particle Accelerator Conference, Knoxville, TN, 2005 (IEEE, Piscataway, NJ, 2005), p. 2059.

45. K. Bane and G. Stupakov, in Proceedings of the Particle Accelerator Conference, Knoxville, TN, 2005 (IEEE, Piscataway, NJ, 2005), p. 3390.

46. K. Bane, G. Stupakov, J. Tu, SLAC/LCLS-TN-05-6, February 2005. 